\title{
Effect of ultrasonic pre-treatment on low temperature acid hydrolysis of oil palm empty fruit bunch
}

\begin{abstract}
Various pre-treatment techniques change the physical and chemical structure of the lignocellulosic biomass and improve hydrolysis rates. The effect of ultrasonic pre-treatment on oil palm empty fruit bunch (OPEFB) fibre prior to acid hydrolysis has been evaluated. The main objective of this study was to determine if ultrasonic pre-treatment could function as a pre-treatment method for the acid hydrolysis of OPEFB fibre at a low temperature and pressure. Hydrolysis at a low temperature was studied using 2\% sulphuric acid; 1:25 solid liquid ratio and $100{ }^{\circ} \mathrm{C}$ operating temperature. A maximum xylose yield of $58 \%$ was achieved when the OPEFB fibre was ultrasonicated at $90 \%$ amplitude for $45 \mathrm{~min}$. In the absence of ultrasonic pre-treatment only $22 \%$ of xylose was obtained. However, no substantial increase of xylose formation was observed for acid hydrolysis at higher temperatures of 120 and $140{ }^{\circ} \mathrm{C}$ on ultrasonicated OPEFB fibre. The samples were then analysed using a scanning electron microscope (SEM) to describe the morphological changes of the OPEFB fibre. The SEM observations show interesting morphological changes within the OPEFB fibre for different acid hydrolysis conditions.
\end{abstract}

Keyword: OPEFB fibre; Dilute acid hydrolysis; Ultrasonic pre-treatment 